\title{
INFRARED LONG BASELINE INTERFEROMETRY
}

\author{
W.C. DANCHI, M. BESTER, P.R. McCULLOUGH and C.H. TOWNES \\ Space Sciences Laboratory, Physics and Astronomy Deptartments \\ University of California at Berkeley \\ Berkeley, CA 94720, U.S.A.
}

During the last few years, two new instruments using long baseline interferometry have been constructed for high angular resolution astronomy in the mid-infrared spectral region (8$12 \mu \mathrm{m})$. One called SOIRDETE-Synthese d'Overture en InfraRouge a DEux TElescopeswas built by J. Gay and his collaborators at CERGA. SOIRDETE has a fixed E-W $15 \mathrm{~m}$ baseline and two $1 \mathrm{~m}$ diameter telescopes of conventional design. This instrument obtains interference fringes by adjusting an optical-precision delay line in discrete steps to compensate for the geometrical delay of the projected baseline. The interference fringe from the source is detected using $\mathrm{HgCdTe}$ photodiodes. Because the instrumental delay has discrete steps a time-domain interferogram is created. This interferogram, upon Fourier transformation to the frequency domain, yields information about the spectral characteristics of the source. First fringes have recently been obtained with this instrument (Gay, 1988).

The other instrument, which uses heterodyne detection of the infrared signal, was built by our group and is called ISI - Infrared Spatial Interferometer. It is constructed from moveable telescopes of a novel design, which allows a number of baselines to be sampled depending on site geometry. ISI has recently obtained its first interference fringes. Because ISI is constructed of telescopes of unusual design, and since its first fringes have not been reported elsewhere, this report gives an overview of its design and initial results.

The ISI telescopes are of Pfund-design and each consists of a $2.03 \mathrm{~m}$ diameter flat mirror that reflects star light to a stationary $1.65 \mathrm{~m}$ diameter parabolic mirror. The flat mirrors sit on alt-az mounts and track the source. Each parabolic mirror brings light to a focus behind the front surface of its flat mirror through a hole in the mirror. The choice of geometry allows a range of $\pm 55^{\circ}$ in azimuthal rotation angle from where the flat mirror points directly at the parabola, and $-2^{\circ}$ to $+55^{\circ}$ in altitude. Unlike conventional alt-az mounts, the Pfund-design has no singularity at the zenith. The flat and parabolic mirrors are mounted in a special semi-trailer. On site, the flat and parabolic mirror mounts rest on kinematic supports sitting on firm, reinforced concrete bases. The trailer bed, which is mechanically decoupled from the mounts, contains an optics room and control room behind the flat mirror. The current site allows for E-W baselines ranging from approximately 4 to $28 \mathrm{~m}$ and N-S baselines up to about $15 \mathrm{~m}$, as well as baselines of intermediate angles (cf. Townes, 1984; Townes et al.,, 1986; Danchi et al.,, 1986).

Infrared and visible light are separated by a dichroic mirror near the prime focus of the parabola. The visible light is imaged on a solid-state MOS camera for acquisition and guiding. The infrared radiation propagates onto an optical table, where it is combined at a beamsplitter with $\mathrm{CO}_{2}$ laser radiation and detected on a $\mathrm{HgCdTe}$ photodiode. This 
heterodyne detection scheme is conceptually similar to the technique used in centimeter and millimeter wavelength interferometry. The theoretical sensitivity of the system is sufficient to allow observation of infrared sources about six magnitudes weaker than the brightest sources. A detailed discussion of the detection system and its sensitivity limits has been reported by Danchi et al., (1988).

After a design and construction phase lasting from October 1983 to December 1987, ISI was moved to Mt. Wilson, in January 1988 and installed with a $4 \mathrm{~m} \mathrm{E}$-W baseline. ISI obtained its first fringes on IRC +10216 , displayed in Fig. 1, on June 29, 1988. To interpret Fig. 1 it is important to know some details about the detection system. The two $\mathrm{CO}_{2}$ laser local oscillators are offset in frequency to take out the normal time variation of the fringe frequency, which results from the changing projected baseline due to the rotation of the earth. As a result, the fringe frequency at the output of the correlator is fixed and, for convenience, we have chosen a value of $10 \mathrm{~Hz}$. Fig. 1 shows a narrow peak at $10 \mathrm{~Hz}$ as expected. The width of the peak is limited by the finite length of the scan to about $0.01 \mathrm{~Hz}$. This peak sits on a broader, shallower peak of approximately $1 \mathrm{~Hz}$ width, which is likely due to atmospheric fluctuations. Further work is necessary to obtain the visibilities of this source and others as well as the spectrum of phase fluctuations due to the atmosphere.

To summarize, the Infrared Spatial Interferometer has just recently obtained its first fringes and should soon begin producing results on many late-type stars, infrared sources embedded in dark molecular clouds, and other interesting astronomical objects.

\section{References}

Danchi, W.C., Arthur, A., Fulton, R., Peck, M., Sutton, E.C., Townes, C.H. and Weitzmann, R.H., 1986, Proc. of the SPIE Conference on Advanced Technology Optical Telescopes $I I I, 628,422$.

Danchi, W.C., Bester, M. and Townes, C.H., 1988, Proc. of the NOAO/ESO Conference on High Resolution Imaging by Interferometry, Ed. F. Merkle, Garching, FRG.

Gay, J., 1988, private communication.

Townes, C.H., 1984, Astrophys, and Astron., 5, 111.

Townes, C.H., Danchi, W.C., Sadoulet, B. and Sutton, E.C., 1986, Proc. of the SPIE Conference on Advanced Technology Optical Telescopes III, 628, 281.

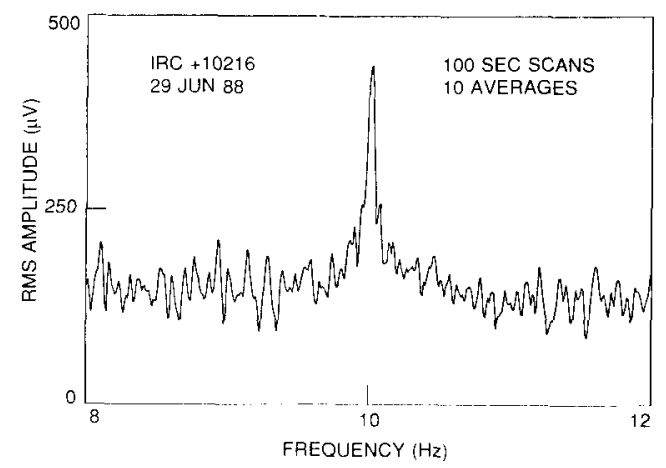

Figure 1. RMS amplitude of an interference fringe as a function of frequency for IRC +10216 . This is the first fringe from an astronomical source obtained by ISI. Note the narrow peak at $10 \mathrm{~Hz}$, which sits on a shallow, broad feature of width about $1 \mathrm{~Hz}$, also centered at $10 \mathrm{~Hz}$. 